\title{
Optimization of the Energy Output of Osmotic Power Plants
}

\author{
Florian Dinger, Tobias Tröndle, and Ulrich Platt \\ Institute of Environmental Physics, Heidelberg University, INF 229, 69120 Heidelberg, Germany \\ Correspondence should be addressed to Florian Dinger; f.dinger@stud.uni-heidelberg.de
}

Received 24 March 2013; Revised 7 June 2013; Accepted 19 June 2013

Academic Editor: Shuhui Li

Copyright (C) 2013 Florian Dinger et al. This is an open access article distributed under the Creative Commons Attribution License, which permits unrestricted use, distribution, and reproduction in any medium, provided the original work is properly cited.

\begin{abstract}
On the way to a completely renewable energy supply, additional alternatives to hydroelectric, wind, and solar power have to be investigated. Osmotic power is such an alternative with a theoretical global annual potential of up to $14400 \mathrm{TWh}$ (70\% of the global electricity consumption of 2008) per year. It utilizes the phenomenon that upon the mixing of fresh water and oceanic salt water (e.g., at a river mouth), around $2.88 \mathrm{MJ}$ of energy per $1 \mathrm{~m}^{3}$ of fresh water is released. Here, we describe a new approach to derive operational parameter settings for osmotic power plants using a pressure exchanger for optimal performance, either with respect to maximum generated power or maximum extracted energy. Up to now, only power optimization is discussed in the literature, but when considering the fresh water supply as a limiting factor, the energy optimization appears as the challenging task.
\end{abstract}

\section{Introduction}

Due to a future lack of fossil fuels and the risk of global climate change caused by $\mathrm{CO}_{2}$ emissions, it appears prudent to aim for an energy supply which is based on renewable energy sources. It appears to be realistic that solar and wind power plants (together with hydroelectric power) will be able to supply the future demand [1]. However, in both cases, the generation depends strongly on natural fluctuations. So additional, renewable base-load capable energy sources are desired. A possible base-load energy source is offered by the use of the mixing entropy when fresh water and salt water (e.g., sea water) are mixed, predominantly at river mouths. In fact, mixing entropy (or rather its reduction) is also derived from solar energy, which drives evaporation from the ocean leading to separation of fresh water and salt water. Its theoretical global annual potential is estimated to be up to 14400 TWh per year [2]. Including economic and ecological boundaries, Statkraft forecasts that in fact only 1600 TWh is usable [3], Zeuner [4] confirms this estimation, which is still $8 \%$ of the global demand in electricity in 2008 [5].

There are several concepts to use mixing entropy for generating electric power; a list is given in [4]. One of them is the concept of "osmotic power," which has been introduced by Sidney Loeb in the seventies; for example, see [6]. Because several components can be adopted from "reverse osmosis" desalination plants, osmotic power plants are the most developed concept to use mixing entropy for generating electric power. In 2009, the Norwegian energy provider Statkraft started operating the first osmotic test power plant [3].

In this paper, the optimal operating salinity level and the optimal operating pressure of an actual, so-called "pressure retarded osmotic" (PRO) power plant using a pressure exchanger are derived. The pressure exchanger is crucial for the constant performance of the power plant but also leads to a significant decrease in efficiency. Its settings and losses are implemented in our model. Aiming for a well-arranged model, we ignored all minor energy losses; for example, we are assuming frictionless flow. Furthermore, the most simple membrane model is applied.

\section{Theory of Osmosis}

When fresh water and salt water are mixed, for example, at a river mouth, the overall entropy increases due to the larger number of possible states for the salt ions. From another point of view, this phenomenon can be explained as an effect which neutralizes the difference in chemical potential. In the case of oceanic salt water, an energy of around $2.88 \mathrm{MJ}$ per $1 \mathrm{~m}^{3}$ of 
fresh water is released when it is mixed with a large amount of salt water. In an osmotic power plant, this energy can be used to generate electric power; in nature, this energy just results in a slight warming of the ocean at the river mouth.

We consider two reservoirs completely filled with water of different salt concentrations. They are in contact via a semipermeable membrane which is permeable for water but not for salt ions. Due to the difference in chemical potential, the system tries to neutralize the difference in the concentration. Because of the near-incompressibility of water, even a small amount of water diffusing from the lower concentration to the higher concentration will cause a large pressure increase on the higher concentration side; the socalled "osmotic pressure" occurs, which is commonly notated by $\Delta \pi$. Its thermodynamic formula is given by van't Hoff's law which reads as follows (with the ion concentration $c_{i}=N_{i} / V$ of the media, the Boltzmann constant $k_{B}$, and the absolute temperature $T$ ):

$$
\Delta \pi=\left(c_{\text {high }}-c_{\text {low }}\right) \cdot k_{B} \cdot T
$$

A macroscopic derivation of the osmotic pressure and van't Hoff's law can be looked up in most textbooks on statistical physics, for example, see [7].

On microscopic scales, the effect of osmosis is still not fully understood, but there are several explanations which work usually with diffusion models. For a list of explanations, see, for example, [8], and for a sample derivation, see [9].

2.1. Permeation. For the flow density $J_{M}$ through a semipermeable membrane holds in the first approximation [10]:

$$
J_{M}=K_{M} \cdot\left(\Delta \pi-p_{\text {counter }}\right) \text {, }
$$

where $K_{M}$ is the permeation coefficient of water, a quantity of the specific membrane, $\Delta \pi$ is the current osmotic pressure (only depending on the concentration difference of the solute), and $p_{\text {counter }}$ is the difference in mechanical pressure.

In a closed system, $p_{\text {counter }}=\Delta \pi$ holds. If there is an outlet at the high concentration side, for example, at the turbine, $p_{\text {counter }}$ is reduced. For later considerations, we define the following:

$$
p_{\text {counter }}:=f \cdot \Delta \pi
$$

where $0 \leq f \leq 1$ is a dimensionless factor which can be controlled externally via, for example, the turbine flow. $f$ is the major parameter in this paper.

For the overall membrane flow $S_{M}$ through a membrane area $A_{M}$ holds:

$$
S_{M}=A_{M} \cdot J_{M}=A_{M} \cdot K_{M} \cdot \Delta \pi \cdot(1-f) .
$$

For the power (= change of mechanical energy per unit time) generated by the flow through the total membrane area $A_{M}$ against the counter pressure results:

$$
P_{M}=p_{\text {counter }} \cdot S_{M}=f \cdot \Delta \pi \cdot S_{M}
$$

\section{Setup of a Pressure-Retarded Osmotic Power Plant}

Figure 1 shows the basic components of a pressure-retarded osmotic (PRO) power plant. The core components are the two water chambers, which are in contact via the semipermeable membrane. The pressure difference between the brackish water and the fresh water is given by $p_{\text {counter }}$, with the higher pressure on the brackish water side. In reality, very large membrane areas are required; therefore, the membranes are packed or coiled in modules in order to save space. With these devices, a packing density of $1000 \mathrm{~m}^{2}$ membrane surface area per $1 \mathrm{~m}^{3}$ module is possible [11]. Membrane modules are already used in desalination plants for decades; so a welldeveloped membrane technology can be adopted for osmotic power plants.

For generating electric power, a turbine is connected to the brackish water chamber. The water flow through the turbine has to be replenished from a fresh water reservoir, for example, a river (upper boxes in Figure 1).

Because the turbine uses brackish water but there is only a fresh water supply, the salt concentration at the brackish water side decreases and therefore the power output of the plant. Aiming for a constant performance, it is necessary to keep the salt concentration of the brackish water constant (and ideally close to the concentration of the salt water reservoir, e.g., the ocean water salinity). This is done by the "pressure exchanger." This device is commercially available and has an efficiency of up to 0.95 [12]. It exchanges a volume of brackish water with, ideally, an equal volume of salt water, by using the mechanical energy of the brackish water volume to pump the salt water volume against the operating pressure in the brackish water chamber. Pressure exchangers are used in desalination plant for decades; up to now, they offer the most efficient way to exchange water volumes of different pressures. Nevertheless, there is an additional pump required to compensate for the pressure loss in the pressure exchanger (lower boxes in Figure 1). Furthermore, on the fresh water side, there is a small amount of flushing required, which is ensured by a fresh water outlet, to avoid buildup of salinity.

3.1. Pressure Exchanger $(P X)$. The efficiency $\eta_{\mathrm{PX}}$ of the pressure exchanger suffers two losses: a fractional loss $\rho_{V}$ in exchanged volume (lubrication) and a fractional loss $\rho_{p}$ (friction) in exchanged pressure [12]. Because there is no mathematical relation stated, we assume both effects to act linearly:

$$
\eta_{\mathrm{PX}}=\frac{E_{\text {mech,salt }}}{E_{\text {mech,brackish }}}=\left(1-\rho_{V}\right) \cdot\left(1-\rho_{p}\right) \text {, }
$$

where $E_{\text {mech }}$ denotes the energy content of the salt, respectively, brackish water. Accordingly, the salt water volume flowing from the high pressure exit of the pressure exchanger to the brackish water volume is a little smaller than the brackish water volume entering the high pressure inlet of the pressure exchanger, $S_{S}=\left(1-\rho_{V}\right) \cdot S_{B}$, and has a little less pressure, $p_{\text {salt }}=\left(1-\rho_{p}\right) \cdot p_{\text {counter }}$. 


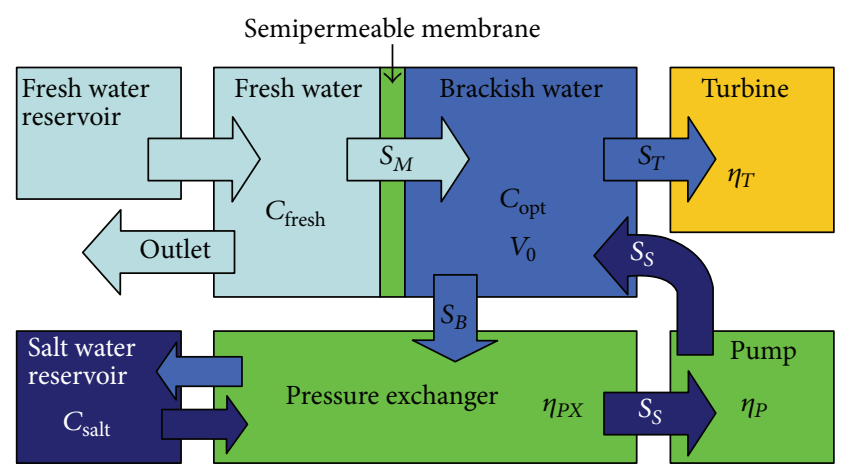

FIGURE 1: Setup of a PRO power plant. Variables in this figure are used later. $S_{M}$ : flow through the membrane; $S_{T}$ : flow through the turbine; $S_{B}$ : flow of brackish water into the pressure exchanger; $S_{S}$ : flow of salt water from the pressure exchanger; efficiency of the: turbine $\eta_{T}$, pump $\eta_{P}$, and pressure exchanger $\eta_{\mathrm{PX}} ; c_{\text {fresh }}$ : salt concentration of the fresh water; $c_{\text {salt }}$ : salt concentration of the salt water; $c_{\infty}$, respectively, $c_{\mathrm{opt}}$ : salt concentration of the brackish water; $V_{0}$ : volume of the brackish water chamber.

The smaller pressure can be corrected by the additional pump. For the required power, $P_{\mathrm{PX}}$ holds (with the power $P_{\text {mech,brackish }}=p_{\text {counter }} \cdot S_{B}$ of the inflowing brackish water flow and the here defined "loss parameter" $\epsilon_{\mathrm{PX}}$ of the whole pressure exchanger system):

$$
\begin{aligned}
P_{\mathrm{PX}} & =\frac{\rho_{p} \cdot p_{\text {counter }} \cdot S_{S}}{\eta_{P}}=\frac{\rho_{p} \cdot\left(1-\rho_{V}\right)}{\eta_{P}} \cdot P_{\text {mech,brackish }} \\
& =\epsilon_{\mathrm{PX}} \cdot P_{\text {mech,brackish }} .
\end{aligned}
$$

The most suitable way to compensate for the reduced volume is a smaller flow through the turbine:

$$
S_{T}=S_{M}-\rho_{V} \cdot S_{B}
$$

3.2. External Control and Optimizations. The flow through the membrane depends on more or less each process parameter and is therefore not directly controllable. Furthermore, the flows of the pressure exchanger depend on each other (and are ideally equal). Thus, the only ways to control the process are the flows at the brackish water inlet of the pressure exchanger and at the turbine. These two process parameters can be set externally and therefore optimized. Via the flow through the pressure exchanger, the salinity of the brackish water and, thus, the overall efficiency of the plant can be optimized (see Section 5). The setting of the turbine flow is determined by the optimal operating counterpressure which depends primarily on the economic boundaries (see Section 6).

3.3. Quantitative Values. For later considerations the following values are assumed:

(i) water temperature: $T=290 \mathrm{~K}$;

(ii) mean ocean salinity: $c_{\text {salt }}=3.5 \%$ salt $(\mathrm{NaCl}$, degree of ionization = 2) $=1196 \mathrm{Mol} / \mathrm{m}^{3}$;

(iii) efficiencies: $\eta_{T}=0.9, \eta_{P}=0.8$, and $\eta_{\mathrm{PX}}=0.94$ (resp., $\left.\rho_{V}=\rho_{p}=0.03\right)$

\section{Salinity and Operating Pressure}

For the process management, it is crucial to control the salt concentration $c(t)$ of the brackish water. The brackish water chamber in a membrane module is very thin, and an operating pressure exchanger leads to turbulence. Thus, the brackish water is assumed to be well mixed and its concentration everywhere is the same. For its changes holds in general:

$$
\frac{d c}{d t}=\lim _{\Delta t \rightarrow 0} \frac{1}{\Delta t}[c(t+\Delta t)-c(t)]
$$

Within the period $\Delta t$, the volumes $\Delta V_{M}, \Delta V_{T}, \Delta V_{B}$, and $\Delta V_{S}$ flow in and out of, respectively, the brackish water chamber. Scaled by the constant volume $V_{0}$ of the brackish water chamber, (9) can be written as

$$
\begin{array}{r}
\frac{d c}{d t}=\lim _{\Delta t \rightarrow 0} \frac{1}{\Delta t}\left[\left(c(t) \cdot \frac{V_{0}-\Delta V_{T}-\Delta V_{B}}{V_{0}}+c_{\text {salt }} \cdot \frac{\Delta V_{S}}{V_{0}}\right.\right. \\
\left.\left.\quad+c_{m} \cdot \frac{\Delta V_{M}}{V_{0}}-A(c(t))\right)-c(t)\right],
\end{array}
$$

where $c_{\text {salt }}$ is the concentration of the salt water and $c_{m}$ is the concentration of the water which is diffusing through the membrane. $A(c(t))$ describes the losses in concentration because ions diffuse through the membrane. For simplification, we assume a perfect semipermeable membrane. This leads on the one hand to $A(c(t))=0$ but also to $c_{m}=0$.

Further, for the volumes hold in general $\Delta V_{i}=$ $\int_{t}^{t+\Delta t} S_{i}(t) d t$. To get the desired condition of a constant performance, the easiest way is to keep also all process parameters, especially the flows $S_{i}$ constant. Accordingly, we from now on set $\Delta V_{i}=S_{i} \cdot \Delta t$. By these assumptions, (10) reduces to

$$
\frac{d c}{d t}=c(t) \cdot \frac{-S_{T}-S_{B}}{V_{0}}+c_{\text {salt }} \cdot \frac{S_{S}}{V_{0}} .
$$

The general solution of this differential equation is given by

$$
c(t)=\left(c(0)-c_{\infty}\right) \cdot \exp \left[-\frac{S_{B}+S_{T}}{V_{0}} t\right]+c_{\infty}
$$

with the steady state concentration

$$
c_{\infty}=\frac{S_{S}}{S_{B}+S_{T}} \cdot c_{\text {salt }} .
$$

Hence, the steady state holds for the osmotic pressure:

$$
\Delta \pi=\left[c_{\infty}-c_{\text {fresh }}\right] \cdot k_{B} \cdot T .
$$

\section{Pressure Exchanger Flow for Optimum Power Plant Efficiency}

In the first approximation, the power plant consists of the power generating turbine and the pressure exchanger plus a pump which costs electric energy. All other technical energy losses are neglected in this paper. 
For the power generated by the turbine $P_{T}=\eta_{T} \cdot p_{\text {counter }}$. $S_{T}$ holds; for the power required by the pressure exchanger system $P_{\mathrm{PX}}=\epsilon_{\mathrm{PX}} \cdot p_{\text {counter }} \cdot S_{B}$ holds. Introducing these two identities as well as (3), (8), (13), and (14), one obtains for the power output $P_{\mathrm{PP}}=P_{T}-P_{\mathrm{PX}}$ of the plant:

$$
\begin{aligned}
P_{\mathrm{PP}}= & f \cdot k_{B} \cdot T \cdot\left(\frac{\left(1-\rho_{V}\right) S_{B}}{\left(1-\rho_{V}\right) S_{B}+S_{M}} \cdot c_{\text {salt }}-c_{\text {fresh }}\right) \\
& \cdot\left[\eta_{T} \cdot S_{M}-\left(\eta_{T} \cdot \rho_{V}+\epsilon_{\mathrm{PX}}\right) \cdot S_{B}\right] .
\end{aligned}
$$

The power $P_{\mathrm{PP}}$ is a function of several constants and the independent process parameters $f, S_{M}, T$, and $S_{B}$. The (economic) factor $f$ and the membrane flow $S_{M}$ will be discussed later. Manipulations of the water temperature $T$ are most probably not favorable; so $T$ is a fixed condition. For getting the maximum power, it is crucial to find the optimal setting for the pressure exchanger flow $S_{B} \cdot\left(d P_{\mathrm{PP}} / d S_{B}\right)=0$ gives (see Figure 2)

$$
S_{B}^{\mathrm{opt}}=\frac{S_{M}}{1-\rho_{V}}\left(\sqrt{1+\phi^{*}}-1\right)
$$

with $\phi^{*}=\left(\left(\left(1-\rho_{V}\right) \eta_{T}\right) /\left(\rho_{V} \eta_{T}+\epsilon_{\mathrm{PX}}\right)\right) \cdot\left(c_{\text {salt }} / \Delta c\right)+\left(c_{\text {fresh }} / \Delta c\right)=$ $\left(\left(\rho_{V} /\left(1-\rho_{V}\right)\right)+\left(\rho_{p} / \eta_{T} \eta_{P}\right)\right)^{-1} \cdot\left(c_{\text {salt }} / \Delta c\right)+\left(c_{\text {fresh }} / \Delta c\right)$ and $\Delta c=c_{\text {salt }}-c_{\text {fresh }}$.

For simplification, we assume from now on $c_{\text {fresh }}=0$ and therefore $\phi^{*}$ reduces to

$$
\phi=\left(\frac{\rho_{V}}{1-\rho_{V}}+\frac{\rho_{p}}{\eta_{T} \eta_{P}}\right)^{-1} \approx 14 .
$$

For the optimal salinity, the optimal operating counterpressure, and the optimal power, we obtain the following results:

$$
\begin{aligned}
& c_{\mathrm{opt}}=\left(1-\frac{1}{\sqrt{1+\phi}}\right) \cdot c_{\mathrm{salt}}, \\
& p_{\mathrm{opt}}=\left(1-\frac{1}{\sqrt{1+\phi}}\right) \cdot f \cdot c_{\mathrm{salt}} \cdot k_{B} \cdot T, \\
& P_{\mathrm{opt}}=f \cdot c_{\mathrm{salt}} \cdot k_{B} \cdot T \cdot S_{M} \cdot\left(1-\frac{1}{\sqrt{1+\phi}}\right) \\
& \cdot\left[\eta_{T}-\left(\eta_{T} \cdot \rho_{V}+\epsilon_{\mathrm{PX}}\right)\left(\frac{\sqrt{1+\phi}-1}{1-\rho_{V}}\right)\right] .
\end{aligned}
$$

Using the values introduced in Section 3 , the extracted electric energy $E_{\mathrm{PP}}$ per volume of fresh water results in

$$
E_{\mathrm{PP}}:=\frac{P_{\mathrm{opt}}}{S_{M}}=f \cdot 1.55 \mathrm{MJ} / \mathrm{m}^{3} .
$$

The theoretical osmotic energy of $1 \mathrm{~m}^{3}$ of fresh water is given by $E_{\mathrm{TD}}=c_{\text {salt }} \cdot k_{B} \cdot T \cdot 1 \mathrm{~m}^{3}=2.88 \mathrm{MJ}$. Therefore, an osmotic power plant using a pressure exchanger (but neglecting all minor losses) has an efficiency of

$$
\eta_{\mathrm{PP}}=\frac{\left(E_{\mathrm{PP}} \cdot 1 \mathrm{~m}^{3}\right)}{E_{\mathrm{TD}}}=f \cdot 0.54 .
$$

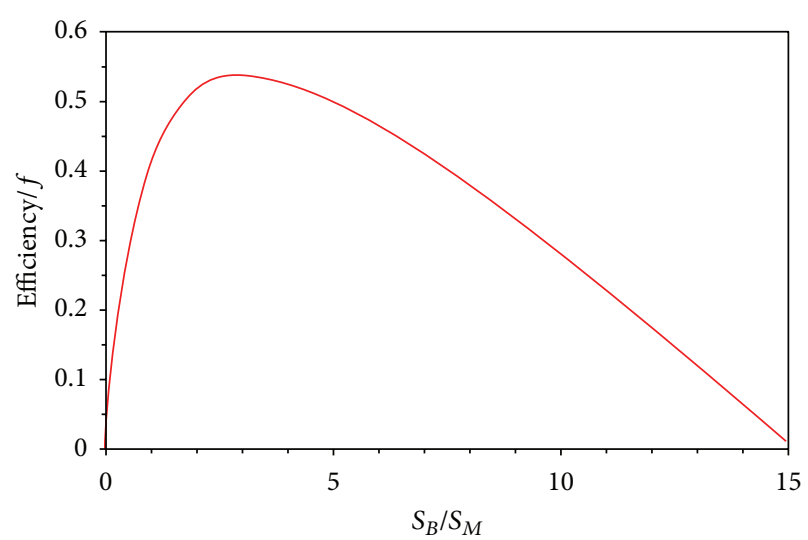

FIGURE 2: The maximum efficiency of the power plant (divided by $f$ ) is plotted as a function of the ratio between $S_{B}$ and $S_{M}$. The optimal efficiency of $f \cdot 0.54$ is reached for $S_{B} \approx 3 \cdot S_{M}$.

\section{Power Optimization versus Energy Optimization}

In this final chapter, the optimal setting of $f$ and therefore of the operating counterpressure is discussed.

From the simple formulae in Section 2, one obtains for the power (= change in mechanical energy per time) through a membrane area $A_{M}$ the following:

$$
P_{M}=p_{\text {counter }} \cdot S_{M}=A_{M} \cdot K_{M} \cdot(\Delta \pi)^{2} \cdot\left(f-f^{2}\right)
$$

which peaks for $f=1 / 2$.

This value is already published and well known as the setting for which the power plant obtains the maximum power output. More distinctly spoken, this factor $1 / 2$ is throughout the literature set "axiomatically" as the optimal value for $f$ (see, e.g., $[10,13])$. This assumption is challenged in the following.

On the other hand, a power plant is frequently not constructed for providing power but electric energy. However, the energy output of a given volume of fresh water depends linearly on $f$ (see (19) and (20)). So the maximum energy output would occur for $f$ near to 1 . Unfortunately, in this limit, the flow and the power per membrane area are very low.

The missing link between these two extreme settings for $f$ is provided by the membrane area $A_{M}$ which can be controlled externally, just by installing the optimal area. So it is possible to go for the energy optimization while still retaining a high power output by providing a larger membrane area. A higher energy output means more revenue but a larger membrane area means also higher investment cost. Hence, finally, $f$ depends purely on economic boundaries. A profit optimization is required for obtaining the economically optimal $f$. In general,

$$
\begin{gathered}
\text { Profit }=\text { Revenue }- \text { Costs, } \\
\text { Revenue }=P_{\mathrm{opt}} \cdot \tau \cdot C_{E}
\end{gathered}
$$

holds with the power output $P_{\text {opt }}$ of the power plant, the lifetime $\tau$ of the power plant, and the energy price $C_{E}$ (in 
matters of generation costs, not corporate profit, given in $€ / \mathrm{kWh})$.

For our purpose, the costs might be split in membrane costs which also include all costs which are directly related to the membrane area like installation and maintenance, and other costs, which are assumed to be not affected by the extent of the overall membrane area:

$$
\begin{aligned}
\text { Costs } & =\text { Membrane costs }+ \text { Other costs } \\
& =A_{M} \cdot C_{M}+\text { Other costs }
\end{aligned}
$$

with the membrane area $A_{M}$ in $\mathrm{m}^{2}$ and the membrane price $C_{M}$ in $€ / \mathrm{m}^{2}$. The $f$ dependency of $A_{M}$ is given by (4):

$$
A_{M}=\frac{S_{M}}{K_{M} \cdot \Delta \pi \cdot(1-f)} .
$$

Using (19) and $E_{\mathrm{PP}}:=f \cdot E_{\mathrm{PP}}^{0}$ for the profit results in

$$
\begin{aligned}
\text { Profit }= & S_{\mathrm{M}} \cdot f \cdot E_{\mathrm{PP}}^{0} \cdot \tau \cdot C_{E} \\
& -\frac{S_{M}}{K_{M} \cdot \Delta \pi \cdot(1-f)} \cdot C_{M}-\text { Other costs, }
\end{aligned}
$$

where $S_{M}$ is fixed by the limiting fresh water supply and not depending on $f$. The maximum profit can be reached with

$$
f_{\max }=1-\sqrt{\frac{C_{M}}{C_{E} \cdot \tau \cdot E_{\mathrm{PP}}^{0} \cdot K_{M} \cdot \Delta \pi}} .
$$

6.1. Visualization of the Optimal $f$ Value. Equation (26) is visualized in Figure 3, where the dependency of $f$ on both economic variables is plotted. $f$ is shown as a function of the energy price in $€$ cent/kWh. Varying membrane prices are indicated by the differently colored branches. Considered are 10,20 , and $40 € / \mathrm{m}^{2}$.

By applying the values introduced above, one obtains $\Delta \pi=21.6$ bar, $E_{\mathrm{PP}}^{0}=1.55 \mathrm{MJ} / \mathrm{m}^{3}$. We assume for the mean lifetime of the membrane (and therefore the whole power plant) $\tau=10$ years. The test power plant operated for 2 years without damage, reported at [3].

The permeation coefficient is usually not published in $\mathrm{m} /\left(\mathrm{s} \cdot \mathrm{Pa}\right.$ ) but in $\mathrm{W} / \mathrm{m}^{2}$ (marked with ${ }^{*}$ ). For our definitions, the value has to be transformed via

$$
K_{M}^{*}\left[\frac{\mathrm{W}}{\mathrm{m}^{2}}\right]=\frac{p_{\text {counter }} \cdot S_{M}}{A_{M}}=K_{M} \cdot(\Delta \pi)^{2} \cdot\left(f-f^{2}\right),
$$

where $K_{M}^{*}$ describes the maximum power density which occurs for $f=1 / 2$ :

$$
K_{M}=\left(\frac{2}{\Delta \pi}\right)^{2} \cdot K_{M}^{*}
$$

The Fraunhofer IGB reported a state-of-the-art value of $K_{M}^{*}=2.3 \mathrm{~W} / \mathrm{m}^{2}$ for lab-intern membranes [14]. For the visualization in Figure 3, we used a value of $2 \mathrm{~W} / \mathrm{m}^{2}$ (full lines) which might be commercially available in the near future. Furthermore, [14] aims for a value of $5 \mathrm{~W} / \mathrm{m}^{2}$. This outlook is also sketched in dashed lines.

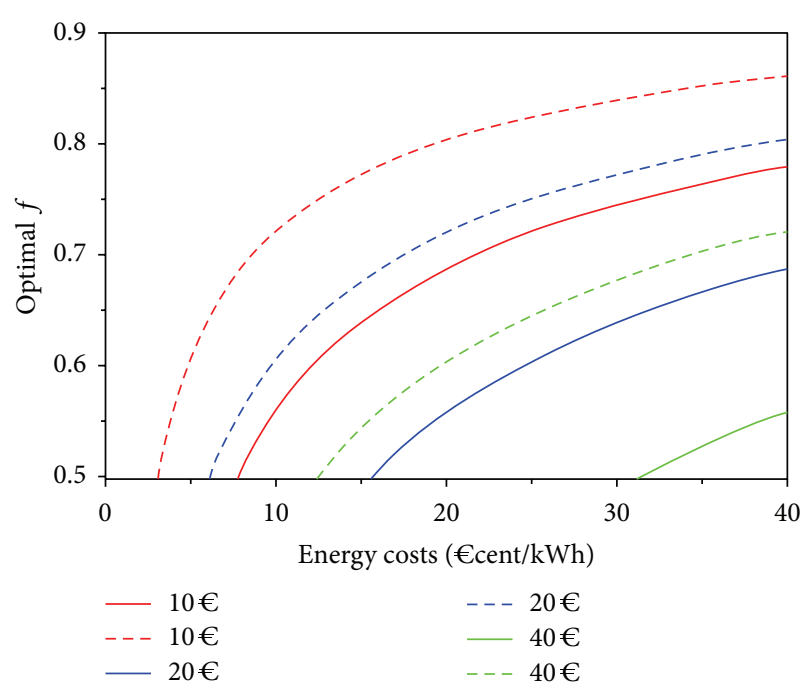

FIgURE 3: The economically optimal value for $f$ as function of the energy price in $€$ cent $/ \mathrm{kWh}$. The different colors indicate different values for the membrane price. Full lines indicate a membrane quality of $2 \mathrm{~W} / \mathrm{m}^{2}$, and dashed lines indicate $5 \mathrm{~W} / \mathrm{m}^{2}$.

\section{Discussion}

The generation costs of solar and wind power are typically of the order of 10-20€cent/kWh, for example, see [15], and the future energy price in an energy system with a higher contribution from renewable energy will probably be close to these values. For this cost range $0.5 \leq f \leq 0.8$ is obtained. So for the current conditions, $f=1 / 2$ appears to be the optimal value. However, on-going research on membranes and largescale production of suitable membranes are likely to bring down membrane cost, while at the same time energy prices are probably rising. Thus, there is no justification to assume this value as the optimal one.

Please be aware that this optimization is just aiming for the maximum profit. If this profit is also positive (and therefore competitive to wind and solar power) depends also on the extent of the Other costs which are not the issue of this paper.

An interesting consequence of the opposing power and energy optimization should be mentioned concerning the degree of capacity utilization. So it seems reasonably to apply energy optimization in general but switching to power optimization (less counterpressure, resp., smaller $f$ ) in times of flooding, for example, in spring. Only rather small additional cost will occur, since only a larger turbine/generator will be required, which constitutes a small fraction of the total cost.

\section{Conclusions}

Two optimizations are performed. In the first step, the optimal salinity was derived by the correct setting of the pressure exchanger. Consequently, for the theoretical efficiency of a pressure-retarded osmotic power plant using a pressure exchanger (and neglecting minor losses), $\eta_{\mathrm{th}}=0.54$ was derived. However, due to economical reasons, the actual 
efficiency of an osmotic power plant is reduced to $\eta_{\mathrm{PP}}=f \cdot \eta_{\mathrm{th}}$, where $f$ is the ratio between the operating counterpressure and the actual osmotic pressure; it is defined $1 / 2 \leq f \leq 1$. In the second step, an estimation for the concrete value of $f$ was derived from an economic optimization. By considering the fresh water supply as a limiting factor and applying a realistic energy price of $10-20 €$ cent/kWh for the coming decades, $0.5 \leq f \leq 0.8$ resulted.

Please be aware that the presented model is condensed to the issues we introduced, that is, a mathematical description for an osmotic power plant using a pressure exchanger and the optimization of its energy output. Higher order membrane effects and technical losses are neglected from the model for retaining clearness. So all quantitative results extracted from this model are overestimated concerning the final energy output. However, implementing these losses would just lead to a more complicated formula for the optimal $f$, but the qualitative results are most likely the same.

The take-away-message of this paper is that there is no justification for the general assumption of a peaking energy output for $f=1 / 2$ which is commonly used in the literature. In fact, a final aim of membrane science appears to be an optimal value for $f$ as near to 1 as possible. The actual value is determined by the quality and costs of the semipermeable membrane and the energy price (of the alternative energy sources). All three quantities are likely to change in the future in favour of osmotic power which means a trend to higher settings for $f$ and therefore to a higher competitiveness of osmotic power.

\section{List of Symbols}

$f: \quad$ Ratio of operating pressure and osmotic pressure

$\Delta \pi: \quad$ Osmotic pressure, $\mathrm{Pa}$

$p_{\text {counter: }}$ : Operating counterpressure, $\mathrm{Pa}$

$p_{\text {opt }}: \quad p_{\text {counter }}$ for optimal settings of the pressure exchanger, $\mathrm{Pa}$

$k_{B}: \quad$ Boltzmann constant, $\mathrm{J} / \mathrm{K}$

$T: \quad$ Absolute temperature, $\mathrm{K}$

$K_{M}$ : Permeation coefficient, $\mathrm{m} / \mathrm{s} / \mathrm{Pa}$

$A_{M}: \quad$ Membrane area, $\mathrm{m}^{2}$

$J_{M}: \quad$ Flow density through membrane, $\mathrm{m} / \mathrm{s}$

$S_{M}$ : $\quad$ Flow through membrane, $\mathrm{m}^{3} / \mathrm{s}$

$S_{T}$ : $\quad$ Flow through turbine, $\mathrm{m}^{3} / \mathrm{s}$

$S_{B}$ : $\quad$ Flow of brackish water through pressure exchanger, $\mathrm{m}^{3} / \mathrm{s}$

$S_{S}$ : $\quad$ Flow of salt water through pressure exchanger, $\mathrm{m}^{3} / \mathrm{s}$

$c_{i}: \quad$ Salt concentration of medium $i, 1 / \mathrm{m}^{3}$

$c_{\infty}$ : Steady state concentration of brackish water, $1 / \mathrm{m}^{3}$

$c_{\text {opt }}: \quad c_{\infty}$ for optimal setting of the pressure exchanger, $1 / \mathrm{m}^{3}$

$V_{0}$ : Volume of the brackish water chamber, $\mathrm{m}^{3}$

$\eta_{T}: \quad$ Efficiency of the turbine

$\eta_{P}: \quad$ Efficiency of the pump
$\eta_{\mathrm{PX}}:$ Efficiency of the pressure exchanger

$\eta_{\mathrm{PP}}$ : Efficiency of the power plant

$\epsilon_{\mathrm{PX}}$ : Loss parameter of the pressure exchanger system

$\rho_{V}: \quad$ Volume loss of the pressure exchanger

$\rho_{p}$ : Pressure loss of the pressure exchanger

$P_{M}$ : Power through membrane, J/s

$P_{\text {opt }}: P_{\text {opt }}$ for optimal settings of pressure exchanger, $\mathrm{J} / \mathrm{s}$

$P_{\mathrm{PX}}$ : Power required for the PX system, J/s

$P_{\mathrm{PP}}$ : Power output of the overall power plant, $\mathrm{J} / \mathrm{s}$

$E_{\mathrm{Pp}}$ : Extracted energy per volume fresh water, $\mathrm{J} / \mathrm{m}^{3}$

$C_{E}: \quad$ Energy price, $€ / \mathrm{kWh}$

$C_{M}:$ Membrane Price, $€ / \mathrm{m}^{2}$

$\tau$ : $\quad$ Lifetime of power plant, $s$.

\section{Acknowledgment}

Florian Dinger wants to thank Werner Aeschbach-Hertig, Institute for Environmental Physics of the University of Heidelberg, for the support during his bachelor thesis.

\section{References}

[1] M. Z. Jacobson and M. A. Delucchi, "Providing all global energy with wind, water, and solar power, part I: technologies, energy resources, quantities and areas of infrastructure, and materials," Energy Policy, vol. 39, no. 3, pp. 1154-1169, 2011.

[2] F. Dinger, Osmosekraftwerke und ihr Potential [Bachelor thesis], Institute for Environmental Physics, University of Heidelberg, 2011.

[3] Statkraft, 2013, http://www.statkraft.com/energy-sources/osmotic-power/.

[4] J. Zeuner, Das weltweite Potential von Mischungsentropiekraftwerken [Bachelor thesis], Institute for Environmental Physics, University of Heidelberg, 2011.

[5] International Energy Agency, 2011, http://www.iea.org/stats/ prodresult.asp?PRODUCT=Electricity/Heat.

[6] S. Loeb and R. S. Norman, "Osmotic power plants," Science, vol. 189, no. 4203, pp. 654-655, 1975.

[7] T. Fliessbach, Statistische Physik, Spektrum, 2010.

[8] F. Borg, What is Osmosis? Explanation and Understanding of a Physical Phenomenon, 2003, http://arxiv.org/ftp/physics/ papers/0305/0305011.pdf.

[9] D. C. Guell and H. Brenner, "Physical mechanism of membrane osmotic phenomena," Industrial and Engineering Chemistry Research, vol. 35, no. 9, pp. 3004-3014, 1996.

[10] A. Achilli, T. Y. Cath, and A. E. Childress, "Power generation with pressure retarded osmosis: an experimental and theoretical investigation," Journal of Membrane Science, vol. 343, no. 1-2, pp. 42-52, 2009.

[11] T. Melin and R. Robert, Membranverfahren, Springer, New York, NY, USA, 2007.

[12] R. L. Stover, "Development of a fourth generation energy recovery device. A 'CTO's Notebook", Desalination, vol. 165, pp. 313-321, 2004.

[13] J. W. Post, J. Veerman, H. V. M. Hamelers et al., "Salinitygradient power: evaluation of pressure-retarded osmosis and 
reverse electrodialysis," Journal of Membrane Science, vol. 288, no. 1-2, pp. 218-230, 2007.

[14] Frauenhofer IGB, Fraunhofer-Institut fuer Grenzaechenund Bioverfahrenstechnik, 2013, http://www.igb.fraunhofer .de/de/kompetenzen/grenzaechentechnik/membranen/osmosemembran.html..

[15] C. Kost and S. C. Thomas, Studie Stromgestehungskosten Erneuerbare Energien, Fraunhofer-Institut fuer solare Energiesysteme, 2010. 


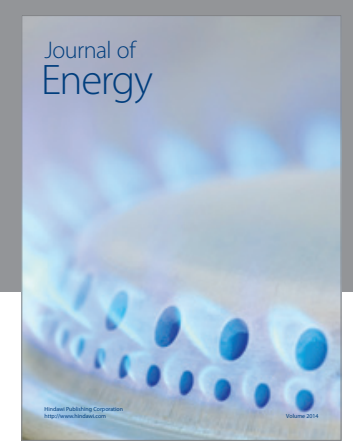

Journal of

Industrial Engineering
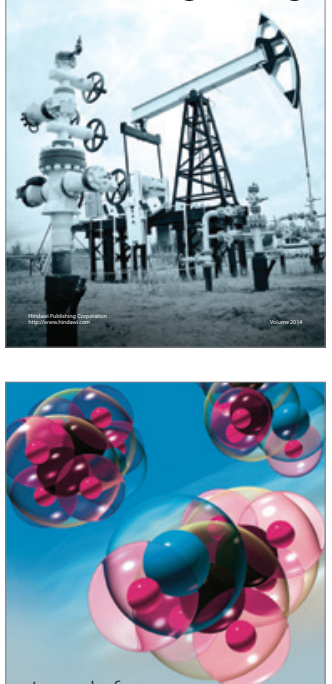

Fuels
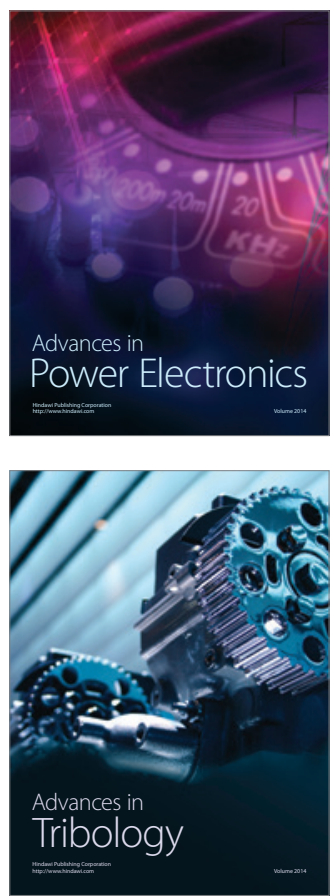

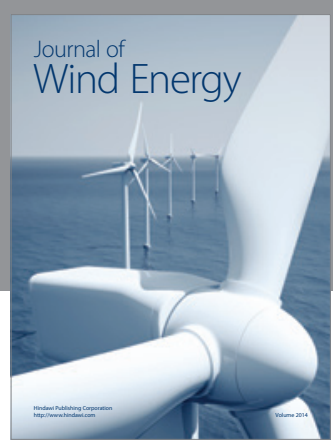

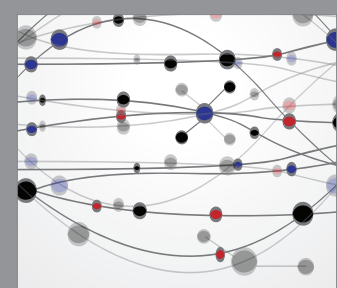

The Scientific World Journal

Submit your manuscripts at http://www.hindawi.com

Journal of

Structures
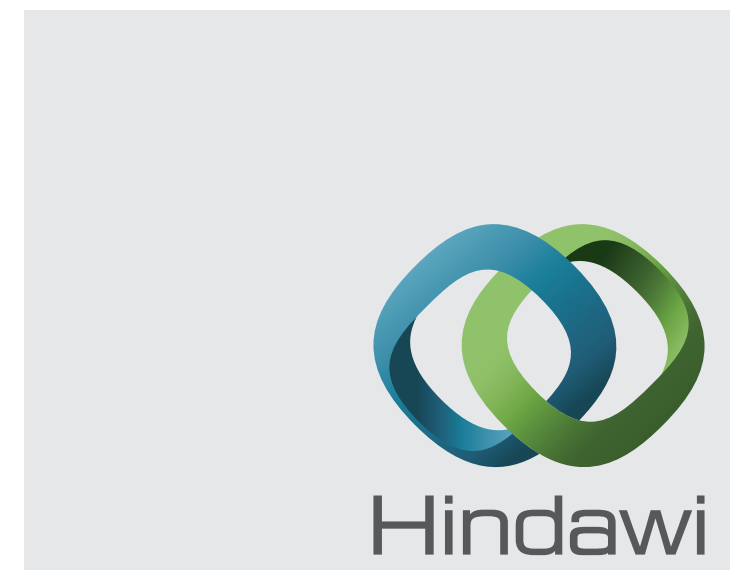

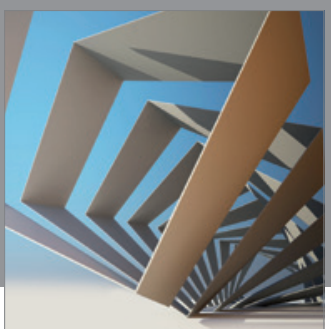

Rotating

Machinery
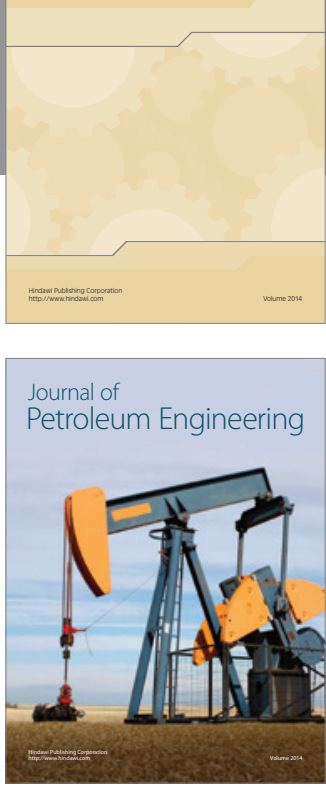

Journal of

Solar Energy
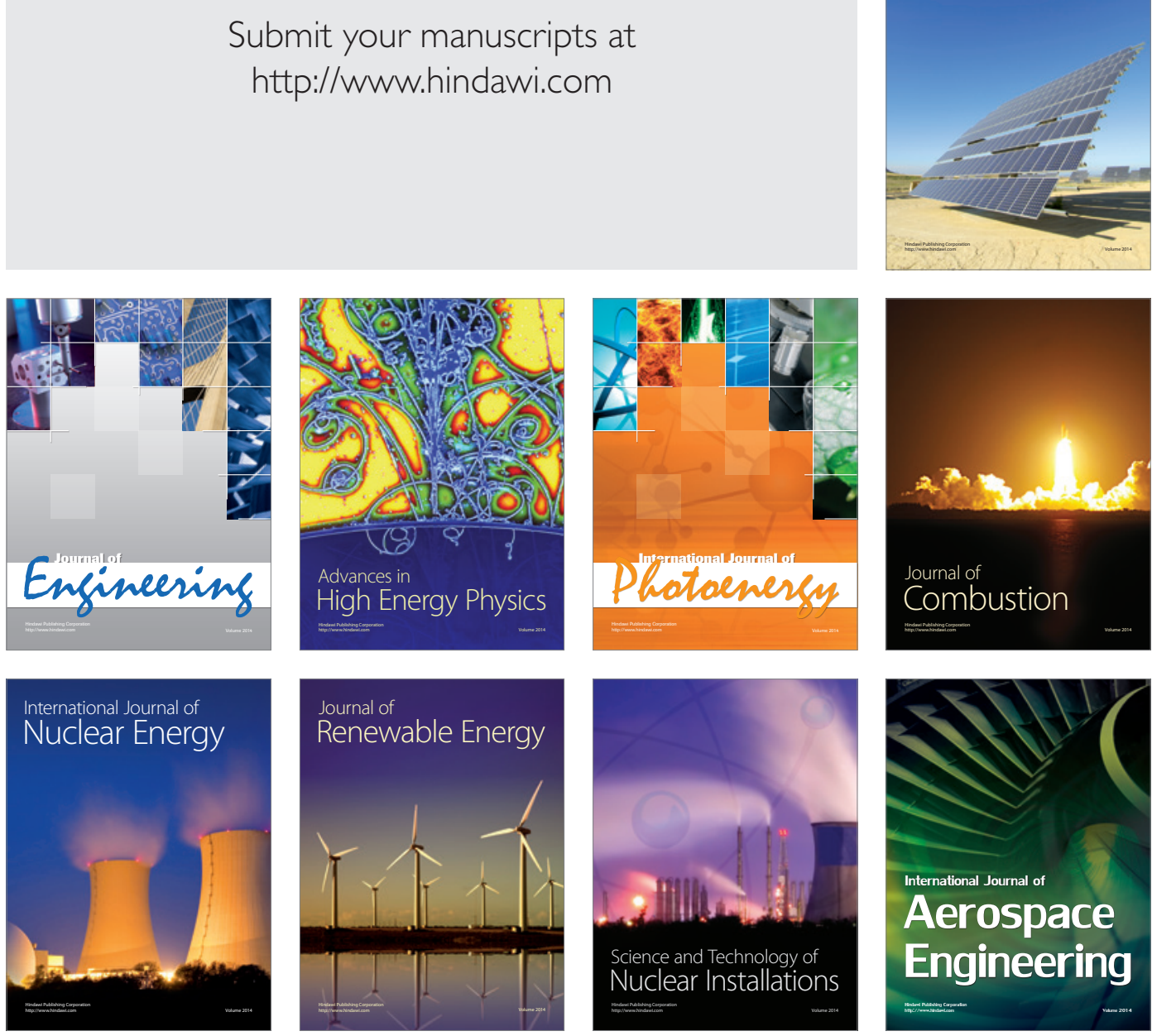